\title{
Montréal : entre transculture et migration
}

\section{Montreal: Between Transculture and Migration}

\author{
Peter Klaus [klauspet@zedat.fu-berlin.de] \\ Freie Universität, Berlin, Allemagne
}

\section{RÉSUMÉ}

Cette conférence plénière pose dès le début quelques sujets de base liés à son titre : migrations, villes, exils. L'argument principal de la conférence plénière consisterait en une comparaison de deux métropoles, Berlin et de Montréal, deux villes de migrations hybrides, multi- et transculturelles. Ces villes occidentales représenteraient des laboratoires, des chantiers où s'expérimentent de nouvelles identités, des identités postmodernes nomades. Lorsqu'à Berlin s'expérimente une nouvelle identité juive, inédite, on pourrait dire qu’à Montréal s'expérimente une nouvelle identité " haïtienne », une nouvelle identité maghrébine, libanaise, ou latino-québécoise. La ville, la métropole ont depuis toujours été des lieux privilégiés de l'hybridité. Elle attire et conserve la différence et favorise en même temps ce glissement vers l'Autre et vers l'Altérité.

\section{MOTS-CLÉS}

Montréal ; Berlin ; migration ; ville ; exil ; identité ; hybridité

\begin{abstract}
The principal theme of this keynote speech consists of a comparison of two metropolis Berlin and Montreal, two cities experiencing hybrid migrations, multicultural and transcultural. These western cities can be viewed as laboratories or "building sites" experimenting with new identities, postmodern nomadic identities. If in Berlin we could speak of experimenting with a new unprecedented Jewish identity, in Montreal we could speak of experimenting with new Haitian, Maghrebian, Lebanese or Latino-Quebecois identities. The city and the metropolis have always been privileged places with regard to "hybridity". They attract and maintain differences while at the same time encouraging a shift towards the Other and Otherness.
\end{abstract}

\section{KEYWORDS}

Montreal; Berlin; migration; city; exile; identity; hybridity

REÇU 2015-08-31 ; ACCEPTE 2016-06-30 


\section{Remarques préliminaires}

Le thème du colloque "(É)Migrations, transferts, exils : métissages et dynamiques de la ville » nous propose un large éventail de réflexions et nous invite à des questionnements qui nous interpellent sûrement tous et toutes, qui nous sommes des passionné(e) s de littérature.

Rien que le fait d'évoquer les notions de «(É)Migration » et d' « exil » nous place devant une problématique qui ne concerne pas seulement la littérature contemporaine, mais davantage encore la question de la survie de millions d'êtres humains de par le monde. Je ne voudrais pas donner dans un catastrophisme facile et de mauvais aloi, mais nous autres, universitaires, chercheurs,etc. ne pourrons pas rester dans notre tour d'ivoire, nous ne pouvons pas rester indifférents devant la misère de ces 50 millions d'êtres humains de par le monde, qui sont en fuite et qui craignent pour leur survie à chaque instant à cause des nombreux conflits qui ravagent actuellement plusieurs régions de la planète.

$\mathrm{Vu}$ les attentats et les tueries insensées de ces derniers temps, nous ne devons pas rester indifférents non plus face à l'ignorance, au fanatisme, à la brutalité et au vandalisme de ceux qui se réclament à tort de l'islam dont ils ne connaissent sûrement même pas le soupçon d'une sourate.

Faisons barrage à l'intolérance et ne permettons pas qu'on sape les fondements de nos valeurs.

\section{Thème du colloque}

Vous avez mis en exergue de ce colloque une citation de Dany Laferrière tirée de son roman L'Énigme du retour (2009), roman qui a mérité à son auteur de nombreuses récompenses, entre autres le Prix Médicis en 2009, et l’an dernier à Berlin (juillet 2014) le Grand Prix International de Littérature. Soit dit en passant, la traductrice allemande a reçu en même temps le prix pour sa traduction du roman vers l'allemand.

On se retrouve ainsi pris dans ce mauvais roman

où domine un dictateur tropical

qui n'arrête pas d'ordonner

que l'on coupe les têtes de ses sujets

à peine a-t-on le temps

de se sauver entre les lignes

vers la marge qui borde la mer des Caraïbes.

Me voilà des années plus tard

Dans une ville enneigée

À marcher sans penser à rien.

Me laissant simplement guider

Par le mouvement de l'air glacial

Et cette nuque fragile qui me précède.

[Dany Laferrière : L'énigme du retour] 
La citation couvre déjà toute la contradiction dans laquelle est catapulté l'émigré/immigré, l'exilé qui se sent ballotté entre deux mondes et confronté à deux univers, le monde menaçant du Sud et le monde du Nord caractérisé par une certaine froideur. Anthony Phelps, le poète haitiano-montréalais, né en 1928 à Port-au-Prince, dans son poème "Frères d'exil » a évoqué cette opposition des deux mondes :

\author{
Frères d'exil \\ compagnons aux pieds poudrés \\ dans nos regards passe une même vision \\ les souvenirs en cage derrière la vitre opaque \\ présent comme une dalle \\ Nous n'avons plus que gestes de fumée \\ pour conter le temps des kénépiers en fleurs \\ car nous entrons dans un domaine étrange \\ de plus en plus tournant le dos au Pays \\ et le verre et l'acier modifient nos croyances \\ Antillais de forte souche et de longue lignée \\ Nous parlons maintenant langage de gratte-ciel \\ Paroles de givre et mots de neige \\ Nous vivons dans une ville \\ où la chanson du remouleur \\ n'est même pas un souvenir \\ où nul ne se rappelle la flûte triangulaire \\ dont les notes aiguës \\ montaient et descendaient le long de notre enfance \\ Nous vivons dans une ville \\ qui jamais ne connut cet homme \\ doué du pouvoir de créer des étoiles \\ en plein midi \\ ville de verre ville d'acier \\ [Anthony Phelps : Frères d'exil ]
}

Les problèmes évoqués dans les deux exemples se ressemblent sans être identiques. Chez Dany Laferrière la fuite et l'exil s'imposent face à la menace d'une mort certaine. Chez Phelps qui fait partie de la génération de ceux qui ont quitté leur pays vers 1964, ce sont les souvenirs en cage, un passé menacé par l'oubli et une acculturation certaine malgré les nostalgiques souvenirs de la chanson du remouleur et le regret d'une perte de la magie de l'enfance dans cette ville de verre ville d'acier.

La ville sans nom est le protagoniste chez Dany Laferrière aussi bien que chez Anthony Phelps, une ville caractérisée et symbolisée par la neige, l'air glacial, par le verre et l'acier. Une ville abri et havre de paix, mais aussi une ville associée à une certaine perte, une absence, un oubli, à une distance. 
Les deux auteurs représentent deux générations d'exilés, d'émigrés ou immigrés qui ont quitté leur pays contre leur gré et qui ont continué dans une ville du Nord dont il sera question plus loin pour se bâtir une nouvelle existence et éventuellement une nouvelle identité. Le dépaysement et l'acculturation qu'ils évoquent dans les extraits cités pourraient être en grande partie généralisés et attribués à un nombre important des écrivains et créateurs issus de l'émigration/ immigration.

Cela dit, depuis toujours, les réfugiés, persécutés et exilés de toutes sortes ont enrichi leurs pays d'accueil avec l'apport de leur différence, de leur altérité. D’une certaine façon nous avons tiré " profit » du malheur de l'Autre, de celui qui a perdu son pays et ses racines et qui a dû se chercher une place, se faire ou se refaire une place, s'approprier une autre langue, une autre culture, se refaire éventuellement une nouvelle identité, une identité métissée.

Les littératures dites «nationales » ont toujours été des carrefours d'idées qui ne se sont pas arrêtées aux frontières nationales. La République Tchèque en est un bel exemple, ayant donné naissance à deux littératures, une en tchèque et une en allemand: pensons à Kafka qui écrit en allemand à Prague et à Milan Kundera, originaire de votre bonne ville de Brnó qui sur le tard a opté pour le français comme langue de création.

Les littératures n’ont jamais été des barrières, bien au contraire. Bien sûr il y a aussi toujours eu des opportunistes parmi les écrivains qui se sont soumis de leur plein gré, par peur ou par lâcheté à certaines idéologies dominantes. Mais c'est une minorité. Les littératures ont toujours été ouvertes aux influences diverses, aux courants novateurs. Elles ont offert l'asile aux imaginaires venus d'ailleurs, et ceci pour le plus grand bien et la plus grande richesse des œuvres et des différents canons ou corpus nationaux. Que serait par exemple la littérature de langue allemande sans Paul Celan (poète français d’origine roumaine et de langue allemande), Elias Canetti (écrivain britannique d’origine bulgare, judéo-espagnole, et de langue allemande), Joseph Roth (écrivain autrichien originaire de la Galicie) ou Manès Sperber (d’origine galicienne, juif, et de culture et de langue allemande). Grâce aux auteurs de la trempe d'un Elias Canetti (Prix Nobel de littérature en 1981) certains chercheurs affirment que l'allemand est de nouveau devenu une langue internationale dans laquelle se produit une littérature universelle, internationale.

Pensons aussi à la ville-carrefour qu'est Trieste, ce pont entre les mondes slaves, romans et germaniques, Trieste la plurilingue et multiethnique ou le Slovène, l'Italien et le Germanophone se cotoient. James Joyce, Rainer Maria Rilke (né à Prague d'ailleurs) et Mark Twain en savent quelque chose. Sherry Simon compare par certains aspects la ville de Montréal à Trieste et aussi à Berlin. Elle y voit une histoire semblable, une ville-frontière qui serait plutôt une ville-pont. Il y a des villes emblématiques qui s'ouvrent à l'Étranger, à l'Autre et qui lui offrent l'occasion d'un nouveau départ. Soit dit en passant que la plupart des nouveaux immigrés et / ou réfugiés se concentrent depuis toujours dans les métropoles, au moins au début, et ceci pour des raisons pratiques, économiques ou sociales. Les nouveaux venus se concentrent surtout là où sont déjà installés des compatriotes. Ça protège et cela rassure.

Dans le Nouveau Monde, c'est New York City avec sa Statue de la Liberté, cette ville emblématique. Combien de milliers ou plutôt millions de réfugiés, d’exilés, de persécutés ont été accueillis à bras ouverts, textuellement et symboliquement parlant. Ils ont par la suite forgé et façonné cette ville, symbole de modernité, de métissage et de potentialité. Aujourd'hui la notion et le concept de «melting-pot » que l'on associe avec New York et les USA est tombée quelque 
peu en désuétude, vu que l'idée même de «melting pot » exclurait le métissage, le plurilinguisme et la multiethnicité.

Certains craignent que le rouleau compresseur et la force assimilatrice de la majorité favorise la ghettoïsation, le côte-à-côte des « Chinatown » ou des « Little Italy » typiques justement pour une métropole « on the move » telle que New York.

Néanmoins, New York a vu naître dans ses murs de nombreux écrivains et écrivaines d'origine les plus diverses (haïtienne, russe, latino). Mais ces écrivains ont abandonné leur langue d'origine, le francais, le russe ou l'espagnol pour ne s'exprimer qu'en anglais.

Ville-carrefour, New York l'est sûrement aussi, mais pas de la même façon que Trieste, la plurilingue ou la ville de Tanger au Maroc d'avant la guerre où se cotoyaient les différentes religions, langues et cultures.

Une publication récente met justement en relief les ressemblances entre New York et Montréal. Dans «Dialogues transculturels dans les Amériques » qui a pour sous-titre " Nouvelles littératures romanes à Montréal et à New York » les auteurs mettent l'accent sur la richesse créatrice résultant des différents flux migratoires et qui donnent souvent des résultats spectaculaires voire innovateurs, tels que l'apport des auteurs hispanophones, entre autres, à New York où même le magazine Time a titré dès 1988 « Magnifico! Hispanic culture breaks out of the Barrio. » À Montréal, il fallait attendre la publication en 1996 du recueil de nouvelles « Les Aurores montréales » de Monique Proulx pour assister à un hommage similaire des auteurs issus de l'immigration. La même année 1996, on a assisté à une polémique controversée autour de l'acceptation de l'écrivain venu d'ailleurs dans le corpus de la littérature québécoise. Monique LaRue a donné voix à cette polémique dans «L'Arpenteur et le Navigateur. » Avant de continuer avec le rôle de Montréal tournons-nous vers Berlin.

Une ville-carrefour qu'il ne faut pas oublier: Berlin où se concentre de nos jours une scène culturelle des plus passionnante. Pensons seulement à l'arrivée et l'implantation d'environ 20.000 jeunes Israéliens, dont de nombreux artistes, qui se sentent plus à l'aise et plus libres à Berlin qu'à Tel Aviv. C'est avec une émotion certaine qu’on observe cet événement, surtout lorsqu'on pense au passé de l’Allemagne et de Berlin.

Pensons aussi à ceux et celles qui façonnent quotidiennement une nouvelle littérature de langue allemande bercée par des rythmes mongols, les chants turcs, kurdes ou arabes. Un métissage nouvelle moûture qui ne va pas toujours sans heurts mais qui est l'image de marque d'une ville en devenir tout comme sa sœur presque jumelle : Montréal. Savez-vous que certains germanistes de par le monde trouvent que l'auteur turco-allemand Zafer Senocak représente au mieux la littérature de langue allemande contemporaine?

Mais que dire de Yade Kara, la turco-berlinoise avec son roman «Selam Berlin » ou de Yoko Tawada, la japonaise qui a élu son domicile dans la langue allemande et à Berlin, pensons au jeune auteur iraquien Abbas Khider qui depuis son arrivée à Berlin en 2000 a publié trois romans en allemand dans lesquels il retrace son passé de prisonnier sous Saddam Hussein. Ce ne sont que quelques exemples, mais qui illustrent la grande diversité et la richesse provenant de ces « Néo-Berlinois » pour utiliser un terme en cours au Québec encore au début des années 1990, les « néos ».

Cette diversité en littérature n'est possible que dans une métropole marquée par l'afflux continuel d'immigrés, de réfugiés, de demandeurs d'asile. Une métropole qui se cherche encore et 
qui se bâtit tous les jours. Pour se faire une idée visuelle de la diversité berlinoise, vous n’aurez qu'à faire le tour de ses lieux de culte et vous allez découvrir aussi bien des églises orthodoxes que des temples hindous, des monastères et temples bouddhistes et des mosquées de différentes obédiences. Ce n'est pas une idylle, c'est un marché des possibles dont l'avantage réside dans l' éventuelle ouverture vers l'Autre d'où qu'il soit.

Je vous ai cité le cas de Berlin, parce qu'il y a des ressemblances avec Montréal bien que la situation montréalaise soit plus complexe encore en littérature.

Mais avant de nous concentrer sur Montréal, jettons d'abord un regard sur la " rivale » canadienne de Montréal. Toronto, la ville-reine. Toronto, tout comme Montréal, a offert asile à de nombreux réfugiés, à des immigrés, à des existences qui ont échoué sur les rives du Lac Ontario.

Pour ceux qui s'intéressent à la littérature en provenance de l'Ontario, il est intéressant de noter qu'un grand écrivain canadien d’origine métissée (srilankais et néerlandaise), Michael Ondaatje, a inversé les rôles dans son roman «In the skin of the Lion » (1987). Dans ce roman, un Canadien de la campagne s'en va à Toronto pour participer à la construction de la ville. Étranger dans sa propre ville, qu'il ne s'est pas encore appropriée, il est accepté et intégré, lui le Canadien, par des ouvriers immigrés qui lui offrent l'asile. Ce thème de l'intégration par l'Autre joue également un rôle dans un nouvelle de Monique Proulx « Les Aurores montréales » (1996). Là, c'est un jeune Montréalais, un ado du nom de Laurel qui se sent perdu et seul dans un environnement qui lui paraît hostile. La solution positive vient de la part de ceux dont il croyait qu'ils étaient ses ennemis, des jeunes issus de l'immigration qui finalement l'intègrent et lui offrent leur amitié. Il est enfin arrivé chez lui.

Mais Toronto ne produit pas seulement une littérature de langue anglaise, la métropole canadienne est loin d'être monolithique, car on y écrit et crée aussi en français. De nombreux immigrés venus d'Afrique sub-saharienne, du Maghreb et d'Europe apportent leur différence linguistique et culturelle en français. Pensons au poète, écrivain et professeur Hédi Bouraoui (York University), originaire de Tunisie, orfèvre de la langue, à Marguerite Andersen, originaire de Berlin et devenue soi-disant une figure de proue d'une nouvelle littérature de langue française, ou pensons à Didier Leclair, cet Africain de Montréal , installé à Toronto et qui a publié il y a quelques années son roman « Toronto, je t'aime» (2001).

\section{Montréal : entre transculture et migration}

J'ai cité ces quelques exemples afin de démontrer que la cible de nos réflexions, la ville de Montréal, n'est pas la seule concernée par la thématique/problématique de notre colloque. J'ai cité ces exemples aussi pour démontrer que toutes ces villes Berlin, Montréal, Toronto ou New York, sont des ensembles urbains inachevés, " on the move », en perpétuel devenir. Berlin l'a montré, surtout depuis la Chute du Mur. Montréal le prouve encore tous les jours.

Ces villes sont des laboratoires, des chantiers où s'expérimentent de nouvelles identités, des identités postmodernes nomades, comme le dit Régine Robin. Lorsqu’à Berlin s'expérimente une nouvelles identité juive, inédite, on pourrait dire qu'à Montréal s'expérimente une nouvelle identité " haïtienne ", une nouvelle identité maghrébine, libanaise, latino-québécoise... entre 
autres. La ville, la métropole, ou la mégapolis (d'après le titre du livre de Régine Robin) ont depuis toujours été des lieux privilégiés de l'hybridité Elle attire et conserve la différence et favorise en même temps ce glissement («imperceptible ») vers l'Autre, vers l'Altérité, c'est ce que certains appellent la «transculture».

\section{Trans, Inter, Multi}

Je ne voudrais pas vous fatiguer avec la discussion concernant la terminologie controversée des différents «trans », « inter » et " multi ». On évoque depuis quelques temps dans de nombreuses publications ces notions qui pour chacun signifient autre chose. Pendant un certain temps la discussion au Québec et au Canada a été dominée d'abord par l'opposition entre "inter » (favorisé par le Québec) et le « multi » (favorisé par Ottawa). C’était une opposition foncièrement politique. Pour faire simple, « multi » signifierait une juxtaposition de " solitudes », le Canada en possédant au moins trois de ces solitudes, de ghettos ethnicisés. Émile Ollivier en dénombre quatre. Lécrivain Neil Bissoondath qui écrit en anglais et vit en français à Québec a fustigé probablement à juste titre les erreurs du multiculturalisme dans son livre « Le marché aux illusions. La méprise du multiculturalisme » où il dit : "One makes a life, puts down roots, and from this feeling of belonging comes the wish to be as fully part of the country as possible. This sentence encapsulates a whole attitude to migration, a way of negotiating the binaries of centre and margin, a resistance of hyphenated identities, that informs all his work. » En Allemagne aussi, le « multiculturalisme » a mauvaise presse, depuis que notre chancelière lui a certifié un échec certain.

"Inter ", par contre, d'après le sens que revêtaient les premières "Semaines de l'Interculturel » au Québec des années 1990, introduirait une sorte d'égalité et de réciprocité dans les échanges entre les différentes cultures.

Une société marquée par l'immigration comme la canadienne se doit de profiter de tout ce que les nouveaux citoyens apportent. La Transculture ou la transculturation selon que l'on privilégie un état ou un processus n'est pas une invention canadienne voire québécoise. Le terme remonte à l'anthropologue Fernando Ortiz Fernandez, le Cubain, qui l'a créé en 1947 en le distinguant justement de l'acculturation dont on a parlé plus haut. Il décrit par ce terme un processus « of merging and converging of different cultures. » Transculturel et transculturation s'appliqueraient à des « identités plurielles », donc des appartenances multiples qui remettraient en question la représentation d'une autonomie ou bien aussi d'une certaine homogénéité. Ces identités plurielles ou appartenances multiples sont le propre d'une société basée sur l'immigration comme celle du Canada. Mais les choses ne sont pas toujours aussi simples. Accepter ou faire accepter des appartenances multiples ne va pas de soi.

Le rôle des «néo-Québécois"

Ce n'est donc pas vraiment étonnant que ce soient justement des Néo-Québécois (on les appelait comme ça encore au début des années 1990), des Italo-Québécois, qui lancent le débat autour de la «transculture » en 1983 en fondant le magazine transculturel «Vice Versa ». Lamberto Tassinari, Antonio d'Alfonso et Fulvio Caccia, ces intellectuels d'origine italienne (seul d'Alfonso est né à Montréal) ont un projet ambitieux avec leur magazine trilingue qui ne vivra que quelques 14-15 ans. 
Mais malgré ce court laps de temps, l'impact de ce projet aura été des plus percutants. Bruno Ramirez, impliqué dès le début dans l'aventure transculturelle de Vice Versa explique la transculture, lui l’enseignant avec une expérience états-unienne et canadienne-anglaise de la façon suivante: «Alors que la transculture - telle que je la vivais - reflétait une réalite quotidienne marquée par la traversée constante de frontières, le multiculturalisme était avant tout une politique étatique... »

Il arrive à un moment propice, à un moment où "La Québécoite » (1983), ce roman polymorphe de Régine Robin a contribué à propulser la littérature québécoise dans la postmodernité. Régine Robin $\left({ }^{\star} 1939\right)$, née à Paris de parents judéo-polonais, est elle-même un bel exemple qui illustre à merveille ce concept de transculturalité, d'identités et d'appartenances multiples.

"La Québécoite », «Vice Versa » et le roman libérateur " Maryse » de Francine Noël marquent cette année charnière 1983.

Une véritable césure d'époque, car rien ne sera plus comme avant.

Depuis une multitude de publications célèbrent le caractère exceptionnel de cet univers littéraire polymorphe que constitue Montréal. Je ne vous cite que quelques titres parus en Europe: "Montréal, mégapole littéraire », tel le titre d' un collectif publié par Madeleine Frédéric en Belgique, "Montréal - Toronto - Stadtkultur und Migration in Literatur, Film und Musik » et Carmen Mata Barreiro qui parle du « Rôle de la littérature migrante dans la construction de la francophonie montréalaise ».

\section{Un petit tour en arrière}

Le Montréal que j'ai connu lors de l'Expo 1967 était encore une ville métaphore des « Deux Solitudes ", ce côte-à-côte des deux communautés anglophone et francophone. Montréal, c’était et c'est d'après les paroles de François Hébert

Métropole d'une minorité au Canada, Montréal pourrait aspirer au titre de la plus importante ville secondaire du monde. Elle est la seconde ville canadienne après Toronto, la seconde ville francophone (si l'on compte les anglophones!) après Paris, la seconde ville juive d'Amérique, la seconde capitale haïtienne. Son équipe de baseball finit généralement au deuxième rang derrière New York...

Montréal, cette éternelle deuxième, devient par la suite au fil des ans la métaphore d'une société canadienne avec toutes ses cassures et l'endroit où se décide le sort de la présence et de la permanence de l'altérité à la française en Amérique du Nord.

Montréal a plus qu'une seule vocation. Montréal est devenu au fils des décennies un havre de paix et d'espoir pour de très nombreux réfugiés. Les différents flux migratoires depuis la fin de la deuxième guerre mondiale ont contribué à façonner le visage et le caractère de Montréal marqué jusque-là essentiellement par ce qu’on a appelé assez longtemps les « Deux Solitudes ».

Entre temps d'autres solitudes se sont greffées sur les premières et ont modifié l'image de Montréal en profondeur. Sherry Simon dans Hybridité culturelle nous parle d'un Montréal hybride de par ses manifestations architecturales. Elle chante l'éloge de Montréal dans son brillant 
essai « Translating Montréal: Episodes in the Life of a Divided City » où elle fait état de cette traversée de la ville par des sensibilités et des créativités autre que simplement « de souche». Et elle dit aussi : "Today Montréal is a cosmopolitan city, with French as the matrix of its cultural life. Its streets are places of contact, mingling, and interference. For almost three centuries, however, the city's cultural relations were described in terms of elemental spatial and cultural divisions. While Montréal has dreams and myths equal to those of any metropolis, its special lessons have to do with those separate pasts. » Les divisions de Montréal évoquées par Sherry Simon semblent être une histoire du passé, l'ancienne "ligne de démarcation », la Main, autrement dit l'avenue Saint-Laurent qui a, semble-t-il, perdu ce rôle historique. Les divisions d'aujourd'hui sont plus subtiles, elles se sont déplacées tout comme les populations " de souche » qui "émigrent » vers les banlieues et celles des immigrées qui ont tendance à se concentrer pour certaines à Montréal-Nord ou à Saint Michel, ou à Outremont.

Sherry Simon évoque aussi la difficile cohabitation des écrivains de "souche » et des écrivains baptisés « anglo-montréalais ». Et pourtant, que serait le destin de Montréal comme creuset de littératures issues d'origines les plus diverses sans les voix anglophones de Mordecai Richler, de Mavis Gallant, de Gail Scott, sans oublier Leonard Cohen, F.R.Scott, Robert Majzels et A.M.Klein, le barde d'un Montréal plurilingue. Ce n'est que sur le tard que la revue «Voix \& Images » a paré à cet oubli il y a quelques années en mettant en valeur l'apport des écrivains montrélais de langue anglaise, dont plusieurs italo-montréalais comme David Fennario et Mary di Michele.

\section{Les Haiitiens}

L'impact le plus sensible a probablement été exercé par la première vague d’immigrés (ou plutôt réfugiés) Haïtiens autour de 1964.

Parmi eux surtout Anthony Phelps, Serge Legagneur, Jean-Richard Forest, Émile Ollivier qui avaient déjà commencé à s'impliquer dans la vie culturelle d'Haïti comme poète, écrivain, animateur, journaliste radio, etc. Contraints à l'exil, ils apportent leur bagage culturel et linguistique, marqué par la diglossie créole-francais, le réalisme merveilleux et d'autres marques perceptibles de l'imaginaire caribéen..

En lisant ces auteurs, il faut avoir en tête qu'il s'agit d'une culture marquée par l'oralité, une dualité linguistique originale que l'exil risque de recouvrir d'une couche d'acculturation.

C’est avec les Haïtiens, d'ailleurs vite intégrés dans les anthologies de la poésie québécoise, que commence la contamination de l'imaginaire québécois par d’autres imaginaires, les Italoquébécois vont suivre avec la publication de différents recueils de textes en prose, de poésies, de pièces de théâtre, et la création de maisons d'éditions etc.

À la différence des Haïtiens, les Italiens, ces immigrés allophones, ont dû s'approprier une autre langue, le français, pour pouvoir participer à la construction et ou façonnement d'une culture et d'une littérature québécois contemporaine. Et Fulvio Caccia, l'écrivain et journaliste italo-québécois qui vit à Paris depuis longtemps a mis l'accent sur l'implication des Autres dans le façonnement du Québec, de sa culture et de sa littérature en disant : «Oui pour un Québec français, mais autrement!» 
C'est cette différence, cet autrement qui importe. Car depuis, de nombreux « autrement » ont dû déferler sur les rives du Saint-Laurent et apporter leur grain de sel.

Les auteurs haitiens ont introduit le réalisme merveilleux caribéen dans la littérature québécoise. Dans leur sillage les divinités du vaudou ont envahi Montréal; des conteurs sans pair comme Émile Ollivier ont bâti des ponts entre le monde du Nord et celui du Sud, p.ex. dans son roman « Passages » (1991/1995) où deux fils narratifs se entrecroisent. Et Gérard Étienne a introduit toute la panoplie haïtienne à Montréal, les désarrois et traumatismes des victimes du duvaliérisme inclus. Son "Ambassadeur macoute " inverse les rôles entre colonisateur et colonisé et développe le projet de se soumettre le monde du Nord avec les moyens des dictatures du Sud. Et si cela ne suffisait pas Gérard Étienne nous présente dans « La Romance en do-mineur de Maître clo » un protagoniste haïtien qui a trouvé refuge à Montréal, mais qui se sent poursuivi par les divinités vaudous, fait qui rend impossible son intégration dans son nouveau pays. Marie-Célie Agnant dans « Le Livre d'Emma » et dans «Un Alligator nommé Rosa » nous démontre que le monde du Nord ne protège pas forcément des méfaits d'une dictature du Sud. Dans « Un Alligator nommé Rosa » elle déterrotorialise les méfaits du duvaliérisme et ses conséquences. La «bataille» décisive entre victime et bourreau aura lieu cette fois-ci dans une sorte de huis-clos non pas à Montréal mais dans le sud de la France, dans la même région qui a offert l'asile à l'ancien dictateur déchu.

Carmen Mata Barreiro parle d'un Montréal comme d'une ville-récit de l'acculturation, de l’engagement et du métissage, une ville qui régorgerait de récits de migrance. Mais n'est-ce pas justement un des atouts de Montréal que d'être tout cela?

Il ne faut pas oublier non plus les auteurs comme Abla Farhoud qui arrive à Montréal avec son Liban natal dans ses bagages et les souvenirs d'une guerre civile interminable ou un jeune auteur dramatique également d’origine libanaise du nom de Wajdi Mouawad qui transforme le théâtre montréalais et lui donne de nouvelles impulsions. Abla Farhoud et Wajdi Mouawad sont des francophones, oui, mais ils sont pour le moins bilingues et biculturels et ils apportent un bagage culturel complexe.

Que dire des milliers d'Algériens qui ont trouvé refuge et une nouvelle patrie à Montréal ? Bashir Bensaddek, né d’origine berbère en Algérie, qui a dû fuir son pays a réalisé une pièce de théâtre du titre de " Montréal la blanche » (2004) (en souvenir d' "Alger la blanche ») où il présente les biographies de nombreux concitoyens malmenés par la guerre civile des années 1990 en Algérie. Grâce à ce travail, il dit avoir redécouvert son algérianité tout en se réclamant du Québec et de Montréal.

Je ne vous parlerai pas ici des autres facettes de la vie culturelle et artistique montrélaise. Mais rien qu'en évoquant l'existence du FTA (Festival Transamériques, autrefois Festival de Théâtre des Amériques), du festival littéraire Metropolis Bleu qui réunit tous les deux ans depuis une douzaine d'années quelques cent auteurs qui s'expriment dans une dizaine de langues, sans oublier une autre réusssite multi-ou transculturelle québécoise " Le Cirque du Soleil », vous montre que Montréal et le Québec n’ont pas fini de nous étonner.

On a évoqué plus haut que le Canada, et Montréal en particulier ont été pendant longtemps symbolisé par les «Deux Solitudes ». Certains prétendent qu'avec la voix des immigrés est arrivée une troisième solitude. Une autre solitude n'est pas mentionné du tout : celle des Amérindiens. Et pourtant, ils élèvent également la voix (textuellement) et grâce aux travaux de Mau- 
rizio Gatti les Québécois sont en mesure de découvrir la richesse du patrimoine amérindien. Ils sont présents avec des romans et nouvelles, des contes, avec des films et du théâtre. Montréal a depuis 1985 un théâtre autochtone « Ondinnok » permanent créé par Yves Sioui Durand et Catherine Joncas.

Émile Ollivier (1940-2002), ce " schizophrène heureux ", commente cet état des choses différemment quant à l'apport et aux rôles de ces voix venues d'ailleurs, en gommant également la voix des Amérindiens. Émile Ollivier se dit appartenir à ceux qui sont les agents culturels de la subversion ou de la subversion culturelle du Québec. Ils contribuent de saper le «national » de l'intérieur. La citation de son roman paru posthumément "La Brûlerie » (2004) précise cette prise de position encore autrement. La Brûlerie compose une sorte de microcosme, un point de rencontre des biographies les plus bigarrées, des personnages qui ne tiennent pas sur place, des nomades qui traînent les tares d'un passé et qui nous présentent dans ce lieu béni un caléidoscope d'imaginaires, de nostalgies et d'amours impossibles. Un monde en perpétuel métissage. Le narrateur dépeint ce Montréal nouvelle mouture vis-à-vis d'un des protagonistes du roman, Dave Folantrain, qui parle de son intention d'écrire un livre sur le Québec des années 1990 :

Je lui dis aussi qu'un vrai travail d'écriture sur Montréal devrait commencer par mettre en scène la parole nomade, la parole migrante, celle de l'entre-deux, celle de nulle-part, celle d'ailleurs ou d'à côté, celle de pas tout à fait d'ici, pas tout à fait d'ailleurs; je lui dis que dans cette ville aux quatre solitudes - celles d'être francophone, anglophone, immigrant et noir -, il faudrait montrer comment notre présence bouscule, bariole, tropicalise le lieu montréalais. (La Brûlerie, 55-56)

Avec cette citation Émile Ollivier nous explique mieux que mille théories ce qui contribue à la particularité montréalaise.

Et lorsqu’on a constaté que la littérature québécoise (qui en fait est une littérature montréalaise) était dominée à ses débuts dans les années 1960-70 par une relative homogénéité, par une sorte de voix monocorde, on est obligé de constater que les écrivains québécois de souche n’ont plus le monopole de dire le Québec.

Lorsque nous associons Berlin à la voix rauque de Hildegard Knef, au Philharmonique de Berlin ou à la musique techno de la Love Parade, et lorsque Paris nous est évoqué par la musique de Debussy et les chansons du poète-anar Léo Ferré, nous vivons Montréal de plus en plus comme une œuvre d'art complexe et inachevée où la littérature et surtout la poésie et le Jazz se réunissent harmonieusement.

Pensons aux nombreux événements littéraires qui ponctuent l'été montréalais, à commencer par le "Mondial de la littérature ", aux lectures accompagnées de musique dans le cadre des "Cinq à souhait » dans un Café de la rue Saint-Denis. Rappelons-nous aussi le poète Gaston Miron qui, accompagné par des musiciens montréalais, à sillonné Montréal et le Québec avec un de ses recueils de poésies «La marche à l'amour.» Pensons aussi aux Tam-Tams, ces signaux musicaux multiculturels qui rythment les week-end aux pieds du Mont-Royal.

La littérature contemporaine québécoise se tourne résolument depuis quelque temps vers le monde et ouvre ainsi la voie d'une littérature Sans Domicile Fixe ou nomade qui œuvre contre l'enfermement dans des tiroirs nationaux. 
Dans ce concert la littérature québécoise voire montréalaise joue toujours le rôle du premier violon, certes, mais elle ne joue plus toute seule. Au moins à Montréal, la contre-basse anglophone se fait de plus en plus entendre et les « voix venues d'ailleurs » sont devenues les nouveaux images de marque de cette polyphonie littéraire.

\section{Références bibliographiques}

Agnant, M.-C. (2004). Le livre d'Emma. Montréal : Éditions du remue-ménage. . (2007). Un Alligator nommé Rosa. Montréal : Éditions du remue-ménage .

Bensaddek, B. (2004). Montréal la blanche. Montréal.

Berger, V., Kirsch, F. P., \& Winkler, D. (2007) (Eds.). Montréal-Toronto-Stadtkultur und Migration. In Literatur, Film und Musik. Berlin : Weidler Buchverlag.

Bissoondath, N. (1994-1995). Le marché aux illusions. La méprise du multiculturalisme. Toronto : Penguin Books. [En anglais: Selling illusions: The Cult of Multiculturalism in Canada.]

Brüske, A., \& Jessen, H. C. (Eds.) (2013). Dialogues transculturels dans les Amériques - Dialogos transculturales en las Américas. Nouvelles littératures littératures romanes à Montréal et à New York. Nuevas literaturas románicas en Montreal y en Nueva York. Tübingen : Narr Verlag.

Étienne, G. (1979). Un Ambassadeur macoute à Montréal. Montréal : Nouvelle Optique.

Farhoud, A. (1998). Le bonheur a la queue glissante. Montréal : Éditions de l'Hexagone.

Frédéric, M. (1991). Montréal, mégapole littéraire. In Actes du Séminaire de Bruxelles (Septembre-Décembre 1991). Bruxelles : Centre d'Études canadiennes. Université Libre de Bruxelles.

Gatti, M. (2004). Littérature amérindienne du Québec. Écrits de langue française. Montréal : HMH.

Hébert, F. (1989). Montréal. Seyssel : Champ Vallon.

Kanagayakam, Ch. (1997). Bissoondath, Neil. In E. Benson, \& W. Toye (Eds.), The Oxford Companion to Canadian Literature (p. 124). Toronto-Oxford-New York : Oxford University Press.

Laferrière, D. (2009). L'Énigme du retour. Paris : Grasset.

LaRue, M. (1996). L’Arpenteur et le Navigateur. Montréal : CETUQ \& Presses de l'Université de Montréal.

MacLennan, H. (1945). Two Solitudes. New York : Duell, Sloan and Pearce.

Mata Barreiro, C. (2005). Rôle de la littérature migrante dans la construction de la francophonie montréalaise. In J. K. Bisanswa, \& M. Tétu (Eds.), Francophonie d'Amérique. Quatre siècles d'échanges Europe-Afrique-Amérique, (Québec, 26-29 mai 2003). Actes du Colloque. Québec : CIDEF-AFI.

Moss, J. (2005). Representing Montréal Algérians on Stage and Screen. Québec Studies, 38, 25-35.

Ollivier, É. (1991/1995). Passages. Montréal: L'Hexagone/Paris : Le Serpent à plumes.

- (2004). La Brûlerie. Montréal : Boréal.

Ondaatje, M. (1987). In the Skin of a Lion. London : MacMillan.

Phelps, A. (1976). Motifs pour le temps saisonnier. Paris : Oswald.

Proulx, M. (1996). Les Aurores montréales. Montréal : Boréal.

Ramirez, B. (2010). À ma façon (A Modo Mio). In F. Cacia, B. Ramirez, \& L. Tassinari (Eds.), La transculture et ViceVersa. Montréal : Les Éditions Tryptique.

Robin, R. (1983/1993). La Québécoite. Montréal : Québec/Amérique: Éditions Typo.

. (2001). Berlin Chantiers. Essais sur les passés fragiles. Paris : Stock. 
Noël, F. (1983/1987). Maryse. Préface de Lise Gauvin. Montréal : vlb éditeur.

Simon, S. (1999). Hybridité culturelle. Montréal : L'Île de la tortue.

. (2006/2008). Translating Montreal. Episodes in the Life of a Divided City. Montreal : McGill Queen's University Press. [Version française: Traverser Montréal. Une histoire culturelle par la traduction. Trad. Pierrot Lambert, nouvelle préface de Sherry Simon, Montréal, Editions Fidés.] 
